\title{
Fourier Analysis of Deformations of Catalytic Gold Nanoparticles under Reaction Conditions
}

\author{
T. Kawasaki***, T. Miura* and T. Tanji***
}

* $\quad$ Dept. Electrical Eng., Nagoya Univ., Furo-cho, Chikusa-ku, Nagoya, 464-8603, Japan

** PRESTO, Japan science and Technology Agency, 4-1-8 Honcho Kawaguchi, Saitama, Japan

*** EcoTopia Science Inst., Nagoya Univ., Furo-cho, Chikusa-ku, Nagoya, 464-8603, Japan

Gold exhibits catalytic activity when it is in the form of fine particles having a size of less than $10 \mathrm{~nm}$ and is tightly supported on specific metal oxides such as $\mathrm{TiO}_{2}$, etc [1,2]. To reveal its mechanism, dynamic observation of the sample structures during the reaction by environmental transmission electron microscope (ETEM) is quite essential. The authors also have developed the closed-type E-cell TEM [3], and had revealed that the shape of catalytic gold nano-particles are deformed during $\mathrm{CO}$ oxidation [4]. In the paper, we report about Fourier analyses of such deformations for the quantitative investigation.

FIG. 1 shows procedure of Fourier analysis of deformations. First, an image of catalytic gold particle taken before starting deformation was selected as a reference (FIG. 1(a)). Next, differential images were calculated between the reference image and the others among a movie during deformations (FIG. 1(b)). Here, value of difference was defined by averaging all absolute values in pixels of each differential image. Time dependency was plotted by calculating value of difference in the images among the movie (FIG. 1(c)). FIG. 2 shows results of time dependencies of deformations in cases of low (0-150 Pa) and high (1000-1250 Pa) pressures of reaction gas $\left(\mathrm{CO}+\mathrm{O}_{2}+\mathrm{N}_{2}\right)$. Dynamical deformations of gold particles supported on $\mathrm{TiO}_{2}$ anatase were observed by TEM H-8000 (Hitachi; 200kV). In this graph, values in the higher gas pressure case are vibrated with larger amplitude and higher frequency, indicating that more marked deformation occurred. FIG. 2(b) and (c) correspond to Fourier transformations of each graph in (a). These data exhibit that the frequency of deformation under the high gas pressure represents almost two times higher than that under the low pressure condition. It should be considered the Fourier analysis of the deformation is the powerful technique as a quantitative evaluation of the particle dynamics and may bring new physical/chemical findings such as periodical phenomena occurred in the catalytic gold nanoparticles.

\section{References}

[1] M. Haruta, Catalysis Today 36 (1997) 153.

[2] S. Giorgio et al., Ultramicroscopy 106 (2006) 503-507.

[3] T. Kawasaki et al., Rev. Sci. Inst. 80 (2009) 113701.

[4] K. Ueda et al., Surf. Interface Anal. 40 (2008) 1725. 

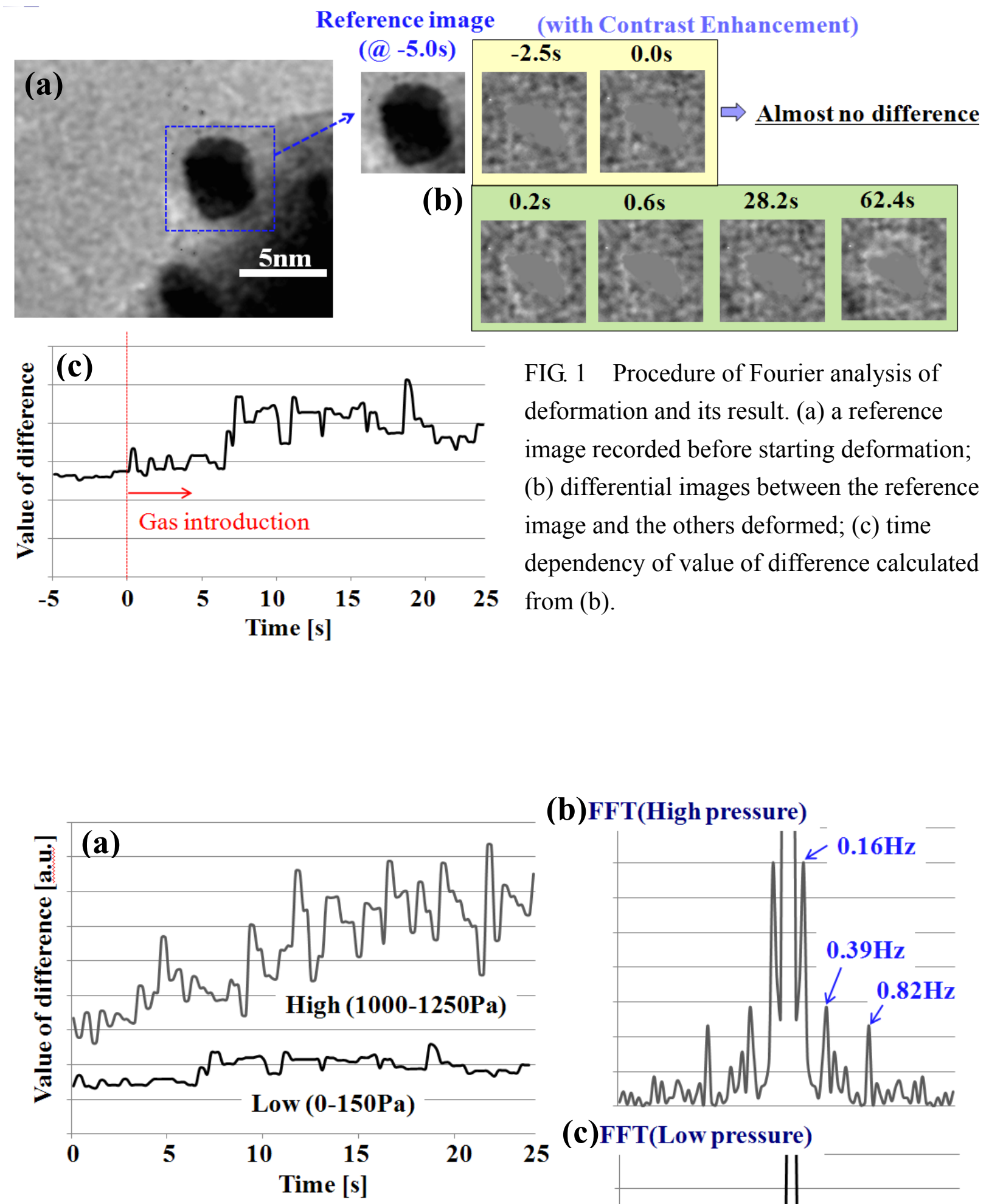

(b)FFT(High pressure)

FIG. 2 (a) Time dependencies of value of difference in cases of high- and low- pressures; (b) Fourier spectrum of high-pressure data in (a), and (c) that of low-pressure data.

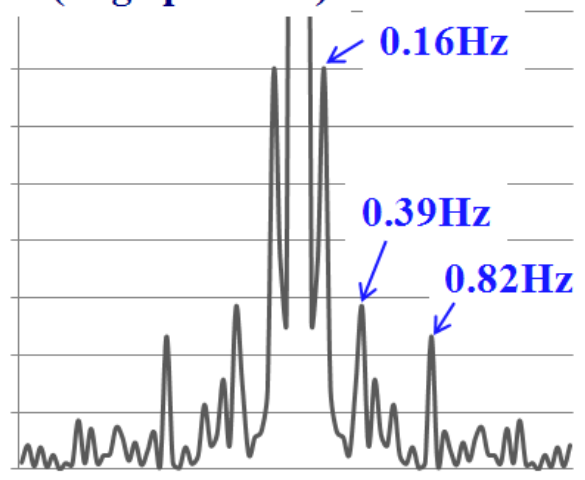

(c)FFT(Low pressure)

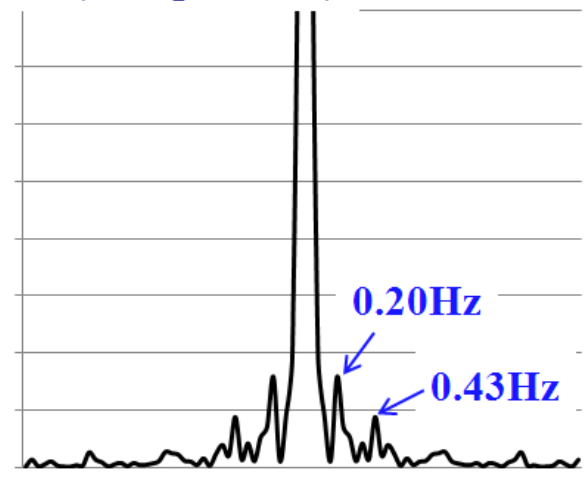

